\title{
Inoculation of Stigma-Colonizing Microbes to Apple Stigmas Alters Microbiome Structure and Reduces the Occurrence of Fire Blight Disease
}

\author{
Zhouqi Cui, ${ }^{1}$ Regan B. Huntley, ${ }^{1}$ Neil P. Schultes, ${ }^{1}$ Blaire Steven, ${ }^{2, \dagger}$ and Quan Zeng ${ }^{1, \dagger}$ \\ ${ }^{1}$ Department of Plant Pathology and Ecology, The Connecticut Agricultural Experiment Station, New Haven, CT, 06511, U.S.A. \\ ${ }^{2}$ Department of Environmental Sciences, The Connecticut Agricultural Experiment Station, New Haven, CT, 06511, U.S.A.
}

Accepted for publication 19 November 2020

\section{ABSTRACT}

Flowers secrete nutrient-rich exudates that support the growth of an assemblage of microorganisms, including both beneficial and pathogenic members, most of which belong to the phylum Proteobacteria. Given the potential role of the microbiome in plant health, manipulating the microbiome to promote growth of beneficial members holds promise in controlling plant diseases. In this study, we inoculated four different bacterial strains that were originally isolated from apple stigmas, alone or in mixtures of increasing complexity, onto apple flowers during bloom. We tested whether such treatments would influence fire blight occurrence, a disease caused by Erwinia amylovora, and whether we could detect a shift in the structure of the microbiome due to the treatments. We show that various inoculations did influence the occurrence of fire blight, although the level of disease suppression was dependent upon specific bacterial strains. Furthermore, treatments using different strains or strain mixtures predominantly resulted in increased representation of the inoculated strains, suggesting that disease suppression was due to an alteration of the stigma microbiome structure. Compared with treatments using single strains, a Pantoea-Pseudomonas strain mixture produced a homogeneous microbiome structure with less interflower variability. Findings from this study suggest that the microbiome on the flower stigma can be manipulated through microbial inoculation. Due to flowers' short life span yet important role in plant disease infection, even a short-term influence on microbiome composition may result in significant decreases in disease susceptibility.

Keywords: bacteriology, fire blight, flower, microbiome, microbiome manipulation, rhizosphere and phyllosphere, stigma
Flowers play a critical role in lifecycles of angiosperm plants by providing a platform for diverse pollination possibilities resulting in the union of sperm with ovules. During pollination, flowers produce nutrient-rich exudates, including stigma exudates, pollen exudates, and nectar, to promote germination of pollen grains or to attract pollinators (Crawford and Yanofsky 2008). Such exudates also support the growth of many microorganisms, including those that

${ }^{\dagger}$ Corresponding authors: Q. Zeng; quan.zeng@ct.gov, and

B. Steven; blaire.steven@ct.gov

Funding: This study was supported by the United States Department of Agriculture-National Institute of Food and Agriculture (USDA-NIFA) Agricultural Microbiome 2020-67013-31794, USDA-NIFA Organic Transitions 2017-5110627001, Northeastern IPM Center Partnership Grant, and USDA-Specialty Crop Block Grant through the State of Connecticut Department of Agriculture.

*The $e$-Xtra logo stands for "electronic extra" and indicates that supplementary figures and supplementary tables are published online.

The author(s) declare no conflict of interest.

(C) 2021 The American Phytopathological Society are either beneficial or pathogenic to the host (Aleklett et al. 2014; Pusey et al. 2009; Spinelli et al. 2005).

Our recent studies characterized the composition and assembly of the microbiome on apple flowers (Steven et al. 2018), with a focus on the stigma (Cui et al. in press). In general, the apple flower microbiome is dominated by bacteria in the phylum Proteobacteria $(>90 \%)$, with small contributions by species of Cyanobacteria, Actinobacteria, Firmicutes, and Bacteroidetes (Steven et al. 2018). Temporal patterns in the stigma microbiome development revealed a diverse bacterial community at the early stage of bloom evolving into a community dominated by two families within the phylum Proteobacteria, the Pseudomonadaceae and Enterobacteriaceae (Cui et al. in press). This suggests that members within the Pseudomonadaceae and Enterobacteriaceae are best adapted for the stigma environment, and form the core members of the apple stigma microbiome (Cui et al. in press; Pusey et al. 2009; Stockwell et al. 2010).

Fire blight, caused by a bacterial pathogen Erwinia amylovora, is an important disease of Rosaceous plants such as apple and pear (van der Zwet et al. 2012). One of the major entry points of E. amylovora to host plants is flowers, and successful infection of flowers is highly dependent on establishing an epiphytic pathogen 
population on the stigma portion of flowers during bloom (Thomson 1986). Stigmatic colonization by E. amylovora is influenced by various biotic and abiotic factors such as temperature, humidity, flower age, and morphology (Pusey 2000; Steiner 1990). To date, no sustainable and completely effective control methods have been developed for this disease, and control mainly relies on the use of copper formulates and the antibiotic streptomycin (Sundin et al. 2016; Vanneste et al. 1995). However, such practices are becoming increasingly limited due to concerns about the phytotoxicity caused by copper and the recent development and spread of streptomycin resistance in E. amylovora populations (McGhee et al. 2011; Sundin et al. 2016). An alternative tool for plant disease management is biological control (biocontrol), the use of a beneficial microorganism to suppress plant disease (Ait Bahadou et al. 2018; Bonaterra et al. 2007; Johnson and Stockwell 1998; Pusey et al. 2009; Sundin et al. 2009; Wilson et al. 1992). In order for these treatments to be effective, the inoculated microbe needs to colonize the host, in essence becoming a member of the microbiome. However, there exists a significant knowledge gap concerning how plant microbiomes are acquired and develop, and how ecological processes such as invasion occur.

There is evidence that microbiome composition can influence plant disease development (Berendsen et al. 2018; Morella et al. 2019; Purahong et al. 2018). A large number of plant pathogens such as Pseudomonas syringae, E. amylovora, and Xanthomonas citri grow epiphytically on plant surfaces prior to causing infection in the plants (Brunings and Gabriel 2003; Pusey 2000; Xin and He 2013). By secreting carbon to the plant surface, plants recruit an assemblage of microbes to compete with the pathogens for nutrients and space, thereby acting as a first line of defense (Leben 1965; Weller et al. 2002). With regards to fire blight control, previous studies have investigated inoculating beneficial bacterial strains to open flowers as biological control (Stockwell et al. 2010, 2011; Sundin et al. 2009). Selection of such strains is often initiated from an in vitro test for antibiosis, or on detached flowers, followed by field testing (Johnson and Stockwell 2000; Pusey 1997). Strains tested for disease control efficacy include P. fluorescens A506, Pantoea vagans C9-1, and Bacillus amyloliquefaciens QST 713 (Ait Bahadou et al. 2018; Chen et al. 2009; Mikiciński et al. 2020; Pusey et al. 2011; Stockwell et al. 2010). Although these treatments have shown promising results in suppressing fire blight disease occurrence and reducing pathogen populations, the downstream effects on the composition of the microbiome have not been assessed.

In this study, we used the apple flower as model to test the hypothesis that manipulating the structure of the microbiome would be associated with changes in disease incidence. Furthermore, we predicted that more complex inoculations consisting of multiple bacterial strains would potentially outperform single-species inoculations by forming a more diverse microbiome structure. Our results demonstrate that inoculating natural stigma-inhabiting bacteria during bloom altered the structure of the stigma microbiome and reduced fire blight disease incidence. However, contrary to our hypothesis, more complex inoculations were not associated with better performance as a biocontrol. Instead, the presence of a specific bacterium, with no observed antagonistic activity, was responsible for decreasing fire blight. Taken together, these data indicate the utility of microbiome manipulation as a tool to control fire blight while also shedding light on factors that control the assembly and composition of the flower microbiome.

\section{MATERIALS AND METHODS}

Sampling site. To exclude the effects of host and environmental conditions, studies were conducted with flowers from 27 trees of the same apple cultivar (Malus domestica 'Golden smoothie') on MM104 rootstock planted in two adjacent blocks at Lockwood Farm, Hamden, CT (41.406 N, 72.906 W) in a 16-foot-by-21-foot spacing. All trees were the same age (planted in 1970) and were maintained under the same management program (https://ag.umass. edu/fruit/publications/2020-new-england-tree-fruit-managementguide). The trees were used in fire blight research in previous years but were subject to extensive pruning to remove the overwinter inoculum prior to the flowering season. During bloom, approximately 300 flowers were produced on each tree. Weather data (temperature and humidity) prior to and during bloom (from 1 to 30 May 2019) is summarized in Supplementary Table S2.

Isolation of stigma colonizing bacteria. Stigma-colonizing bacteria were isolated from the stigma portion of Early Macoun apple flowers at full bloom on 8 May 2018. The stigma portion of an single apple flower was dissected and placed in a sterile $1.5-\mathrm{ml}$ microcentrifuge tube containing $200 \mu \mathrm{l}$ of $0.5 \times$ phosphate-buffered saline (PBS). In total, 100 single flowers from six trees were sampled. The microcentrifuge tubes containing stigma were placed in a water bath sonicator for $5 \mathrm{~min}$ followed by $30 \mathrm{~s}$ of vortexing. PBS $(5 \mu \mathrm{l})$ from each tube was spread on Lysogen Broth (LB) agar plates containing cycloheximide $\left(50 \mu \mathrm{g} \mathrm{ml}^{-1}\right)$ to inhibit fungal growth. Single colonies of different morphologies from each plate were restreaked onto a fresh LB agar plate to obtain pure cultures. More than 1,000 bacterial isolates were obtained. The $16 \mathrm{~S}$ ribosomal RNA (rRNA) of the isolates was PCR amplified using $63 \mathrm{f}$ (5'-CAG GCC TAA CAC ATG CAA GTC-3') and 1387r (5'-GGG CGG WGT GTA CAA GGC- $\left.3^{\prime}\right)$ and sequenced by Sanger sequencing. The 88 strains described in this study make up a representative subset based on the strains' colony morphology.

Bacterial treatments of open flowers. Bacterial strains of Pseudomonas sp. strain CT-1059, Pantoea sp. strain CT-1039, Enterobacter sp. strain CT-1341, and Curtobacterium sp. strain CT- 1342 were stored at $-80^{\circ} \mathrm{C}$ in $20 \%$ glycerol. All strains were cultured in $\mathrm{LB}$ medium at $28^{\circ} \mathrm{C}$ overnight and adjusted to an optical density at $600 \mathrm{~nm}\left(\mathrm{OD}_{600}\right)=1.0\left(\right.$ approximately $\left.10^{9} \mathrm{CFU} / \mathrm{ml}\right)$. The 27 apple trees were randomly labeled into nine treatment groups, with 3 replicate trees in each treatment group. Each treatment group received with one of nine treatments: (i) Pseudomonas, (ii) Pantoea, (iii) Enterobacter, (iv) Curtobacterium, (v) PseudomonasPantoea, (vi) Pseudomonas-Pantoea-Enterobacter, (vii) PseudomonasPantoea-Enterobacter-Curtobacterium, (viii) water, and (ix) streptomycin. Microbial treatments, regardless of single strain or strain mixtures, contained equal amount of the total bacteria $(4 \times$ $\left.10^{7} \mathrm{CFU} / \mathrm{ml}\right)$. Overnight bacterial culture $(600 \mathrm{ml}$, adjusting to $\mathrm{OD}_{600}=1.0$ ) was directly diluted into 14.2 liters of water and used for spray. Equal amounts of ingredient bacteria were mixed together at the site of application. Water was used as a negative control.

Microbial and water treatments were applied twice, once at $50 \%$ bloom (8 May 2019) and again at 70\% bloom (9 May). Approximately 3.8 liters of bacterial suspension at the final concentration of $4 \times 10^{7} \mathrm{CFU} / \mathrm{ml}$ was applied to each tree. E. amylovora was inoculated onto the flowers by spraying a bacterial suspension $(1 \times$ $\left.10^{6} \mathrm{CFU} / \mathrm{ml}\right)$ on 12 May (100\% bloom), immediately after sampling of stigma microbiome. The antibiotic streptomycin (100 ppm) was applied on the same day (12 May), $2 \mathrm{~h}$ after E. amylovora inoculation, and again at $24 \mathrm{~h}$ after the first inoculation.

Sampling for the stigma microbiome and quantification of disease incidence. Sampling of stigma microbiomes was performed on 12 May 2019 at $100 \%$ bloom, 3 days after the second microbial treatments. Ten flowers were collected from a labeled branch of each treated tree, from which the unopened flowers were removed prior to microbial treatment to ensure that all flowers received the inoculum. The stigma portion of each individual flower was 
harvested with a sterile scissors and placed in a sterile $1.5-\mathrm{ml}$ microcentrifuge tube. Collected samples were kept on ice during transportation and were stored at $-80^{\circ} \mathrm{C}$ until DNA isolation.

Three weeks after pathogen inoculation (30 May), inoculated flowers were rated for the development of blossom blight symptoms (black withering, dying of the apple flowers, and emergence of ooze droplets). Percentage of diseased flower clusters in the total number of flower clusters was determined for each tree (Johnson and Temple 2013). For each individual tree, disease symptoms were rated on more than 200 flower clusters by three individual people.

DNA extraction and 16S rRNA gene sequencing. DNA was extracted from the surface of stigmas collected from each individual flower. Stigmas were submerged in $200 \mu l$ of $0.5 \times$ PBS buffer in a microcentrifuge tube. Epiphytic microbes were removed from stigma surface by water bath sonication for $5 \mathrm{~min}$, followed by vortex for 30 s. Stigmas were removed from the tube and the DNA was extracted from the solution using the DNeasy PowerSoil Pro Kit (Qiagen, Hilden, Germany) according to the manufacturer's instructions. The isolated DNA in $100 \mu \mathrm{l}$ of water was further concentrated by precipitating in $100 \%$ ethanol. Isolated DNA was used as template in a PCR to amplify the V4 region of the bacterial $16 \mathrm{~S}$ rRNA gene using the 515f/806r primer set, with both primers containing a 6-bp barcode unique to each sample (Sinclair et al. 2015). The amount of template DNA added in the PCR $(25 \mu \mathrm{l})$ ranged from 10.0 to $20.0 \mathrm{ng}$, as determined by Nanodrop2000 (Thermo Fisher Scientific, Waltham, MA, U.S.A.). Peptide nucleic acid clamps to block the PCR amplification of apple plastid and mitochondrial sequences were included in the reaction, as previously described by Steven et al. (2018). Successful PCR amplifications at the correct amplicon size were confirmed by gel electrophoresis. The PCR products were purified and normalized using the SequalPrep normalization plate kit (Invitrogen, Carlsbad, CA, U.S.A.). Pyrosequencing was conducted on an Illumina MiSeq platform through services provided by the Yale Center for Genome Analysis.

Bioinformatics and statistical analysis. Illumina sequencing reads were assembled into contigs and quality screened using mothur v1.39.5 (Schloss et al. 2009). Sequences that were at least 253 bp in length, contained no ambiguous bases, and no homopolymers of more than 8 bp were used in the analysis. Chimeric sequences were identified by using the VSEARCH (Rognes et al. 2016) as implemented in mothur, and all potentially chimeric sequences were removed. To maintain a similar sampling effort between samples, samples with $<10,000$ sequences per sample were also removed. The resulting sequence counts per sample are presented in Supplementary Table S1. Negative control (PCR using sterile $\mathrm{H}_{2} \mathrm{O}$ as a template) was also included in sequence datasets. The sequence data are deposited at the Sequence Read Archive under accession number PRJNA628946.

Sampling effort was normalized to the depth of the smallest sample and operational taxonomic units (OTUs) were defined at $100 \%$ sequence identity, employing the OptiClust algorithm in mothur (Westcott and Schloss 2017). Taxonomic classification of sequences was performed with the Ribosomal Database Project classifier against the SILVA v132 reference alignment in mothur (Quast et al. 2012; Wang et al. 2007). Nonmetric multidimensional scaling (NMDS) was used to visualize the pairwise distances among samples with Bray-Curtis distances in the Vegan package in $\mathrm{R}$ (Dixon 2003). Descriptive diversity statistics were calculated in mothur. The nonparametric Shannon's diversity index and disease incidence in each treatment were generated with the ggplot2.0 package for R (Wickham 2016). Statistically significant differences in diversity statistics were identified with a one-way analysis of variance and the Tukey-Kramer post hoc test in the agricolae package in $\mathrm{R}$.
In vitro coinoculation assay and antagonistic assay. Overnight LB cultures of Pseudomonas sp. strain CT-1059, Pantoea sp. strain CT-1039, Enterobacter sp. strain CT-1341, and Curtobacterium sp. strain CT-1342 were adjusted to $\mathrm{OD}_{600}=1.0$ (approximately $10^{8}$ $\mathrm{CFU} / \mathrm{ml})$. A narrow line of each strain inoculum was streaked on both horizontal and perpendicular orientations on the LB agar plate using an inoculum loop. For the antagonistic assay, a testing strain was inoculated at the center of an LB agar plate. After $48 \mathrm{~h}$ postincubation, E. amylovora strain 110 was stamped onto the plates using a prong dipped in bacterial suspension (approximately $10^{2} \mathrm{CFU} / \mathrm{ml}$ ). Each treatment had three biological replicates and the experiment was repeated twice.

\section{RESULTS}

Selection of strains for microbiome manipulation. A culture collection of 88 bacterial strains was obtained from the stigmas of apple flowers of cultivar Early Macoun collected from Lockwood Farm, CT (41.406 N, $72.906 \mathrm{~W})$. 16S rRNA gene sequencing placed the isolates in three phyla and five families (Supplementary Fig. S1). We chose four strains for testing their efficacy in suppressing fire blight disease (Table 1; Supplementary Fig. S1B). The rationale for choosing these four strains was as follows. Pseudomonas and Pantoea were chosen because there is a history of using related strains in fire blight control (Stockwell et al. 2010, 2011). Genus Enterobacter was chosen because it was closely related to a highly abundant OTU found in our previous work (Cui et al. in press). We anticipated that it might inhibit pathogen colonization by niche exclusion, by effectively monopolizing the stigma environment. Finally, genus Curtobacterium was selected as an out-group. Our previous data showed that bacteria within the phylum Proteobacteria generally accounted for $>90 \%$ of the microbial diversity of the stigma (Cui et al. in press). We wanted to test whether a bacterium outside this phylum could colonize the stigma and observe the interaction with disease development.

We also wanted to design more complex strain mixtures in order to test whether increasing the complexity of the inoculated strains would affect disease suppression. A coinoculation assay demonstrated that the strains tested did not antagonize each other when cultured under an in vitro condition (Supplementary Fig. S2). We employed Pseudomonas and Pantoea spp. as the base strains for the mixtures based upon the previous use of related strains in fire blight biocontrol (Stockwell et al. 2010). The strain mixtures increased in complexity from two to four strains, with equal representation of each component strain (mix 1, Pseudomonas and Pantoea; mix 2, Pseudomonas, Pantoea, and Enterobacter; mix 3, Pseudomonas, Pantoea, Enterobacter, and Curtobacteria).

Impact of microbiome manipulation on fire blight disease occurrence. The microbial inoculum consisted of overnight cultures diluted to a final density of $4 \times 10^{7} \mathrm{CFU} \mathrm{ml}{ }^{-1}$. Water was used as a negative control. The seven inoculations, including four singlestrain treatments and three strain mixtures, were spray inoculated onto open flowers of apple trees twice during the period of bloom at the point at which approximately 50 and $70 \%$ of flowers were observed to be open. To evaluate whether the microbial treatments had any impact on the occurrence of fire blight disease, we inoculated the pathogen E. amylovora to flowers 4 days after the initial microbial treatments. The antibiotic streptomycin was used as a positive control treatment, and was sprayed to flowers twice, at 2 and $24 \mathrm{~h}$ post pathogen inoculation. The percentage of flowers that developed disease symptoms was assessed 3 weeks later. In total, $33.2 \%$ of water-treated and $11.7 \%$ of streptomycin-treated flowers developed fire blight symptoms (Fig. 1). Compared with the water treatment, flowers that received a treatment of any single or multiple 
strains showed a trend toward lower disease incidence, although the difference was only significant for the Pantoea-alone treatment and the various strain mixtures (Fig. 1). None of the treatments achieved the same disease reduction as the streptomycin treatment, yet the Pseudomonas-Pantoea and the four-strain mixture produced statistically similar results (Fig. 1). Among the single-microbe treatments, flowers treated with Pseudomonas or Pantoea showed lower disease incidence than those treated with Enterobacter and Curtobacterium, though the Pseudomonas treatment was not significantly different than the water control (Fig. 1). Compared with the single-strain treatments, the strain mixtures did reduce disease incidence; however, they were not significantly different than Pantoea alone (Fig. 1). These data indicate that Pantoea may be the core contributor to the control of fire blight on apple flowers, although its activity may be partially enhanced with the addition of other flower microbiome members.

Microbiome composition after manipulation. To determine whether the inoculated microbes influenced the stigma microbiome, we performed 16S rRNA gene sequencing to evaluate the microbiome composition of each individual flower sample 3 days after the second microbial treatment (right before pathogen inoculation). In total, 11,610,049 high-quality filtered sequences were obtained from 78 samples, with the number of sequences ranging from 68,767 to 190,579 (Supplementary Table S1). These sequences were clustered into 147,102 OTUs (mean 2,823 per sample) at $100 \%$ sequence similarity.

The Bray-Curtis distances among samples of each microbial treatment were visualized using NMDS, which showed that samples of the same microbial treatment clustered together and separated from samples from other treatments (permutation analysis of variance, $P=0.001$ ) (Fig. 2A). This result was confirmed by $\beta$-disper test $(P=0.002)$ and suggests that the inoculated microbes did indeed affect the microbiome structure. Samples of the control dataset (water-treated) showed a large dispersion, suggesting a high variation of microbiome structure among individual flowers under natural conditions (Fig. 2A). Among different single-strain treatments, a higher level of variation in the microbiome structure among individual flowers was observed in Pseudomonas (Fig. 2A, red) and Curtobacterium (Fig. 2A, yellow) treatments than the Pantoea (Fig. 2A, green) and Enterobacter (Fig. 2A, light blue) treatments, suggesting that the impact of microbial manipulation on the microbiome structure is largely strain dependent. The structures of microbiome manipulated by mixtures of three (Fig. 2A, dark blue) and four (Fig. 2A, purple) microbes largely overlapped, suggesting that addition of multiple strains drove the structure of the microbiome to a similar composition. Notably, a consistent observation was that the treatments that included the Pantoea strain showed tighter clustering between samples, suggesting a smaller variability in microbiome structure between individual stigmas (Fig. 2A).

Diversity of the stigma communities was assessed by calculating the Shannon's diversity index. Samples of different single-strain treatments displayed various levels of diversity, with the diversity in Enterobacter-treated samples being the lowest in comparison with others. In samples treated with double-, triple-, and quadruple-strain mixtures, we observed an increase in microbial diversity as the number of strains included in the treatment increased (Fig. 2B). Differences in diversity were further supported by the similar patterns in community richness and evenness (Fig. 2B, lower panels). These findings suggest that inoculation of different strains

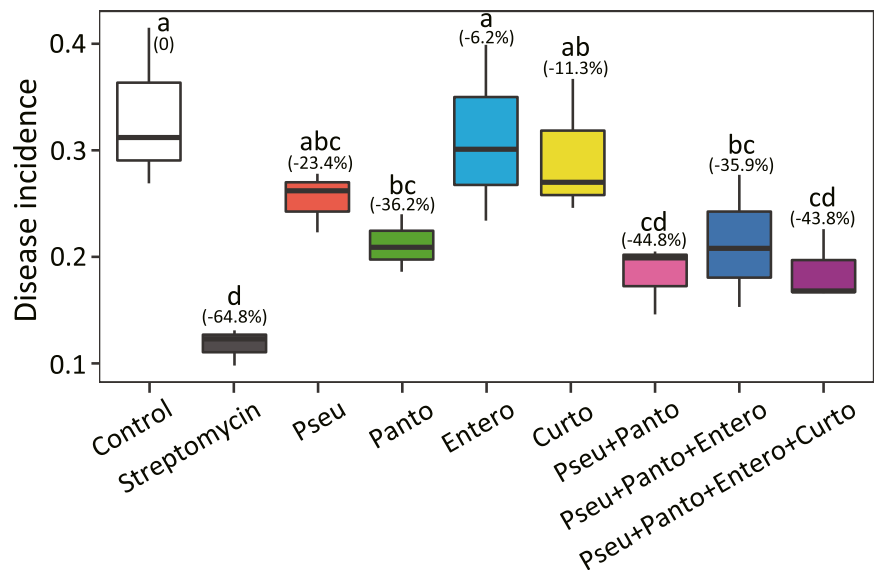

Fig. 1. Percentage of apple flower clusters that developed fire blight symptoms. Bacterial treatments were applied at 50 and $70 \%$ bloom, with three individual apple trees included in each treatment. Erwinia amylovora strain 110 was applied to all trees at $100 \%$ bloom 3 days after bacterial inoculations. Change in disease incidence compared with the water-treated control are indicated in parentheses. Different letters denote statistical significance $(P<0.05$, identified by analysis of variance comparisons of means, employing a posthoc Tukey-Cramer test for multiple comparisons). Control: flowers sprayed with $\mathrm{H}_{2} \mathrm{O}$, Streptomycin: flowers sprayed with antibiotic streptomycin (100 ppm), Pseu: Pseudomonas spp., Panto: Pantoea spp., Entero: Enterobacter spp., and Curto: Curtobacterium spp.

TABLE 1

Taxonomic assignments of stigma-isolated bacteria used for this study ${ }^{a}$

\begin{tabular}{|c|c|c|c|c|c|}
\hline \multirow[b]{2}{*}{ Isolates } & \multicolumn{2}{|l|}{ Blastn by NCBI } & \multicolumn{2}{|c|}{$\begin{array}{l}\text { Relationship to } \\
\text { representative OTU } \\
\text { sequences }\end{array}$} & \multirow[b]{2}{*}{ Accession $^{d}$} \\
\hline & Closest BLAST match & $\mathrm{SI}(\%)^{\mathrm{b}}$ & Match $^{\mathrm{c}}$ & $\mathrm{SI}(\%)^{\mathrm{b}}$ & \\
\hline Pseudomonas spp. CT-1059 & Pseudomonas graminis & 100 & OTU2 & 100 & MT396635 \\
\hline Pantoea spp. CT-1039 & Pantoea agglomerans & 100 & OTU3 & 100 & MT396714 \\
\hline Enterobacter spp. CT-1341 & Enterobacter spp. & 100 & OTU1 & 100 & MT396725 \\
\hline \multicolumn{6}{|c|}{$\begin{array}{l}\text { a Operational taxonomic unit (OTU) numbers: OTU1 = Enterobactersp. strain CT-1341, OTU2 = Pseudomonas sp. strain CT-1059, OTU3 = Pantoea } \\
\text { sp. strain CT-1039, and OTU4 = Curtobacterium sp. strain CT-1342. } \\
\text { b Sequence identity. } \\
\text { c Closest match. } \\
\text { d Accession number. }\end{array}$} \\
\hline
\end{tabular}


and strain mixtures to flowers affected the flower microbiome, resulting in different community structures and diversity.

Phylum level taxonomic bins and the Enterobacteriaceae and Pseudomonadaceae ratio. To investigate how the microbial inoculations affected the composition of the stigma microbiome, $16 \mathrm{~S}$ rRNA gene sequences were classified to the phylum level. In total, 24 phyla were detected across the different treatments. Excluding the Curtobacterium treatment, samples were composed predominantly of Proteobacteria ( 94.1 to $99.9 \%$ ), with a small percentage of Actinobacteria (0.1 to 5.9\%). This included the water control, with no bacterial treatment (Fig. 3A). In the samples manipulated with genus Curtobacterium, a member of the Actinobacteria, the percentage of Proteobacteria was reduced to a mean of $53.8 \%$ (Fig. $3 \mathrm{~A})$. Looking at the ratio of Actinobacteria sequences in each of the flowers treated with Curtobacterium spp., a wide variation between flowers was observed, with Proteobacteria sequences still being dominant in several of the individual samples (Fig. 3B). Moreover, samples manipulated with the four-microbe mixture also exhibited a slight decrease in percentage of Proteobacteria, which could be explained by the colonization of Curtobacterium (Fig. 3A). These findings indicated that, although Curtobacterium is not typically an abundant core member of the stigma microbiome, it is still able to predominate on stigmas when given a competitive advantage by artificial inoculation.

We have previously shown that two families of bacteria tend to predominate on the stigma, the Enteorbacteriaceae and the Pseudomonadaceae (Cui et al. in press; Steven et al. 2018). We determined the ratio of Enteorbacteriaceae to Pseudomonadaceae $\left(\mathrm{R}_{E / P}\right)$ of individual flowers across the treatments. The $\mathrm{R}_{E / P}$ was reshaped by the various microbiome manipulations. The $\mathrm{R}_{E / P}$ of the water-treated dataset varied significantly (243\%) among the samples (Fig. 3C). Some microbial treatments still displayed high variations in the $\mathrm{R}_{E / P}$ (such as the single-strain treatments of Pseudomonas and Curtobacterium), yet many other treatments tended to stabilize the $\mathrm{R}_{E / P}$ between individuals, as observed in single-strain treatments of Pantoea and Enterobacter and all strain mixture treatments (Fig. 3C). The effect was most pronounced in the single-strain treatments of Pantoea and Enterobacter, which became almost exclusively dominated by the family Enteorbacteriaceae to the exclusion of the family Pseudomonadaceae (Fig. 3C). Interestingly, the Pseudomonas treatment did not result in a similar exclusion of Enteorbacteriaceae, suggesting that Pseudomonas is not as adept at monopolizing the stigma environment. Similarly, the multiple-strain mixtures that included Pseudomonas
A

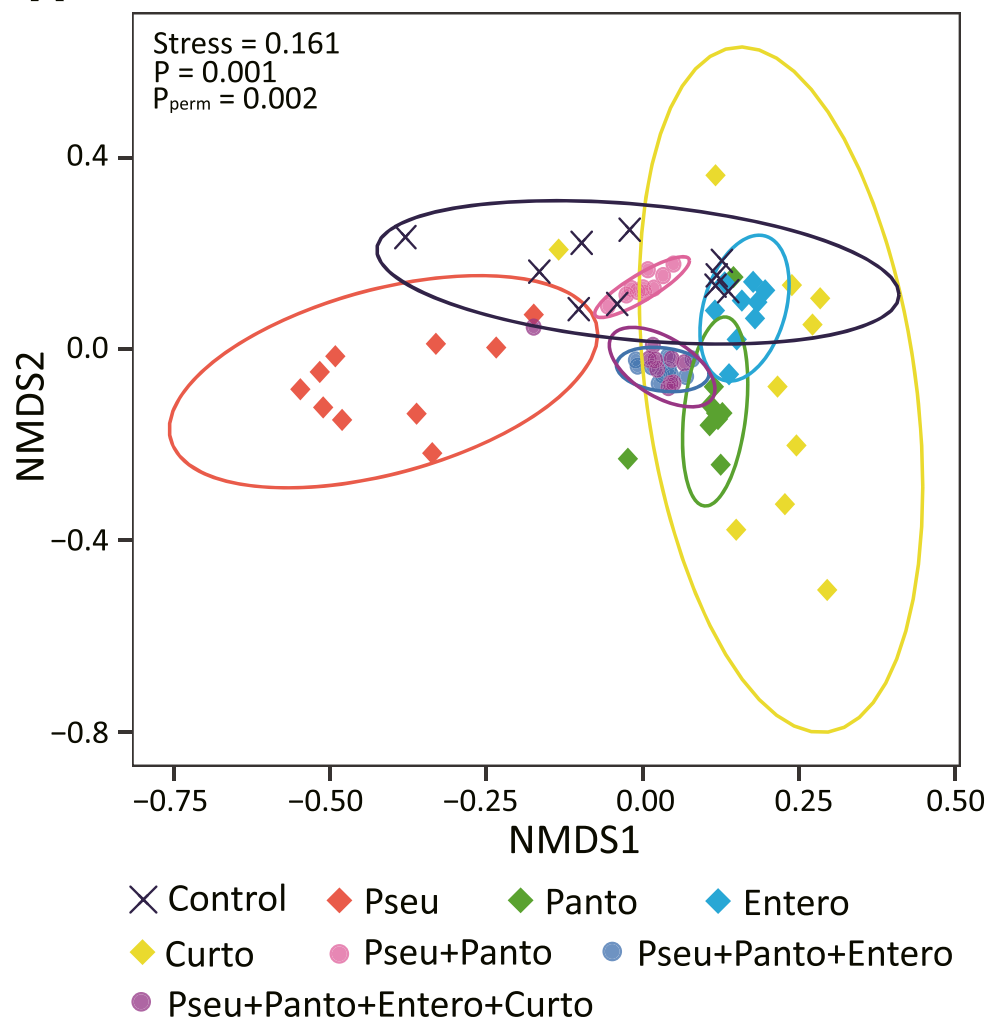

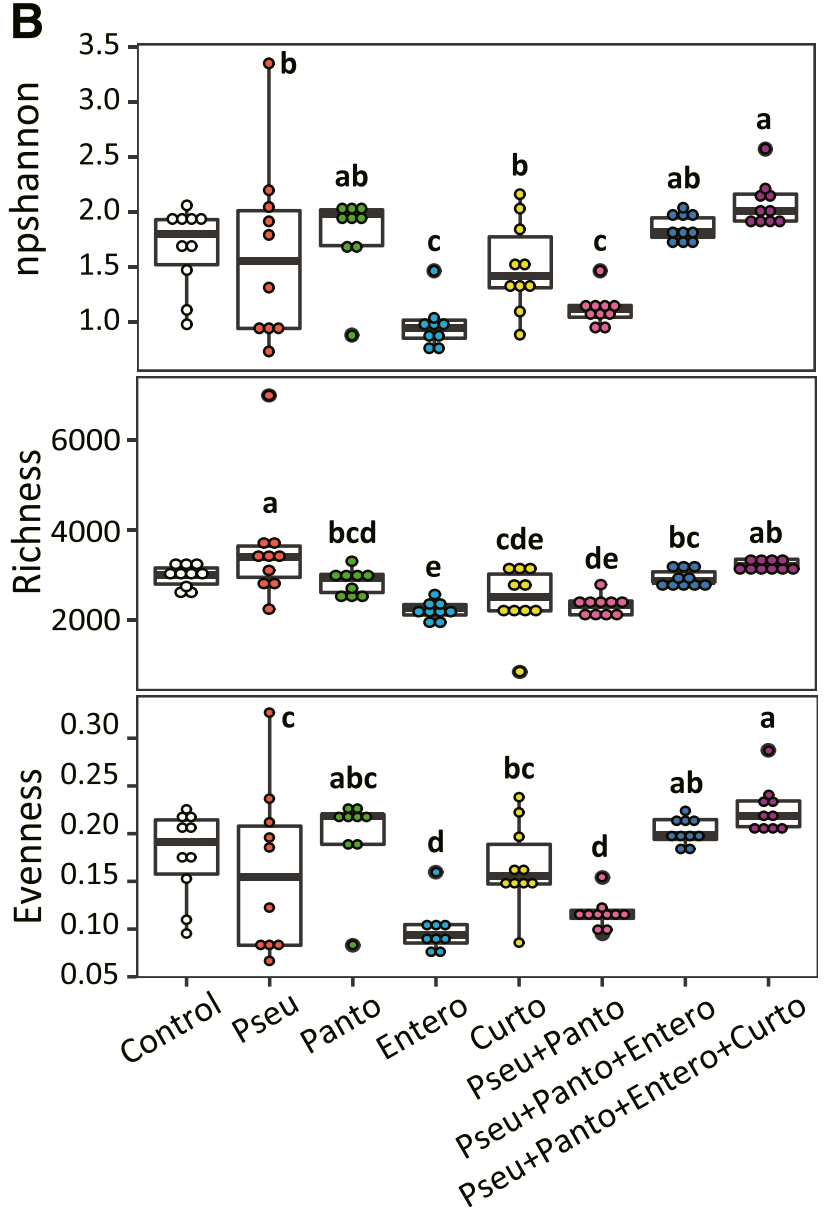

Fig. 2. A, Nonmetric multidimensional scaling (NMDS) plot displaying relationships of stigma microbial community composition in samples from watertreated control (black X) and different bacterial treatments. Distances were calculated using the Bray-Curtis metric and the stress value of the ordination is indicated. Statistically significant differences in clustering were evaluated via both permutation analysis of variance and the $\beta$-disper test, and $P$ values (95\% confidence) are indicated. B, Comparative analysis of community diversity (Shannon index), richness (number of recovered operational taxonomic units), and evenness (Shannon's evenness) among stigma samples. Different letters denote statistically significant differences $(P$ value $<0.05)$ identified by analysis of variance comparisons of means, employing a posthoc Tukey-Cramer test for multiple comparisons. Control datasets are included for reference only and were not included in the statistical comparisons. Control: flowers sprayed with sterile $\mathrm{H}_{2} \mathrm{O}, \mathrm{Pseu}$ Pseudomonas spp., Panto: Pantoea spp., Entero: Enterobacter spp., and Curto: Curtobacterium spp. 
consistently showed a higher ratio of Enteorbacteriaceae, suggesting that Enterobacteriaceae consistently outcompetes Pseudomonadaceae and achieves higher relative abundances.

Identification of the inoculated strains in the microbiome. The sequences in each dataset were classified to the OTU level $(100 \%$ sequence identity) and representative sequences for each OTU were interrogated against the isolate sequences. Four OTUs were identified that shared $100 \%$ sequence identity to the isolates: OTU1Enterobacter, OTU2-Pseudomonas, OTU3-Pantoea, and OTU4Curtobacterium. The relative abundance of each OTU was employed as a metric to determine the isolate's abundance in the stigma microbiome. OTU1 was the most abundant and conserved in the dataset (relative abundance ranging from 0.6 to $94.2 \%$ of recovered sequences), followed by OTU2 (0 to $94.9 \%$ ), OTU3 (0 to $72.2 \%$ ), and OTU4 (0 to $92.1 \%$ ). In the water-treated control samples, the majority of OTUs were accounted for by OTU1 (mean $62.2 \%$ ) and OTU2 (mean 6.7\%), with a large abundance of other OTUs (rare $=31.1 \%$ ) (Fig. 4). OTU abundance was generally predictable from the treatments, in that the inoculated OTUs were enriched in the treatment datasets (Fig. 4). There were two notable exceptions. OTU1-Enterobacter was highly abundant whether or not it was included in the inoculation. OTU1 showed the lowest abundance when it was coinoculated with Pseudomonas. However, including other bacteria in the inoculation once again allowed OTU1 to dominate the stigma microbial community. The second exception to an inoculated strain gaining an advantage in the microbiome was OTU3-Pantoea. When inoculated alone, it achieved a mean relative abundance of $42.0 \%$ yet, when coinoculated with Pseudomonas, it only accounted for $0.04 \%$ of sequences. However, the mean relative abundance of Pseudomonas in the Pseudomonas-Pantoea mixture (9.6\%) was significantly lower than when Pseudomonas was sprayed alone (75.4\%). Moreover, the coinoculation of Pseudomonas and Pantoea together resulted in a relative abundance of OTU1-Enterobacter similar to when Enterobacter was inoculated alone. This apparent paradox of coinoculating Pseudomonas and Pantoea benefitting the noninoculated Enterobacter may point to a competition between two organisms opening a niche for a third. Taken together, these observations point to complex interactions in the plant microbiome that underpin our need to characterize the functional ecology of this system, and documenting the cooperative and antagonistic relationships between community members.
A
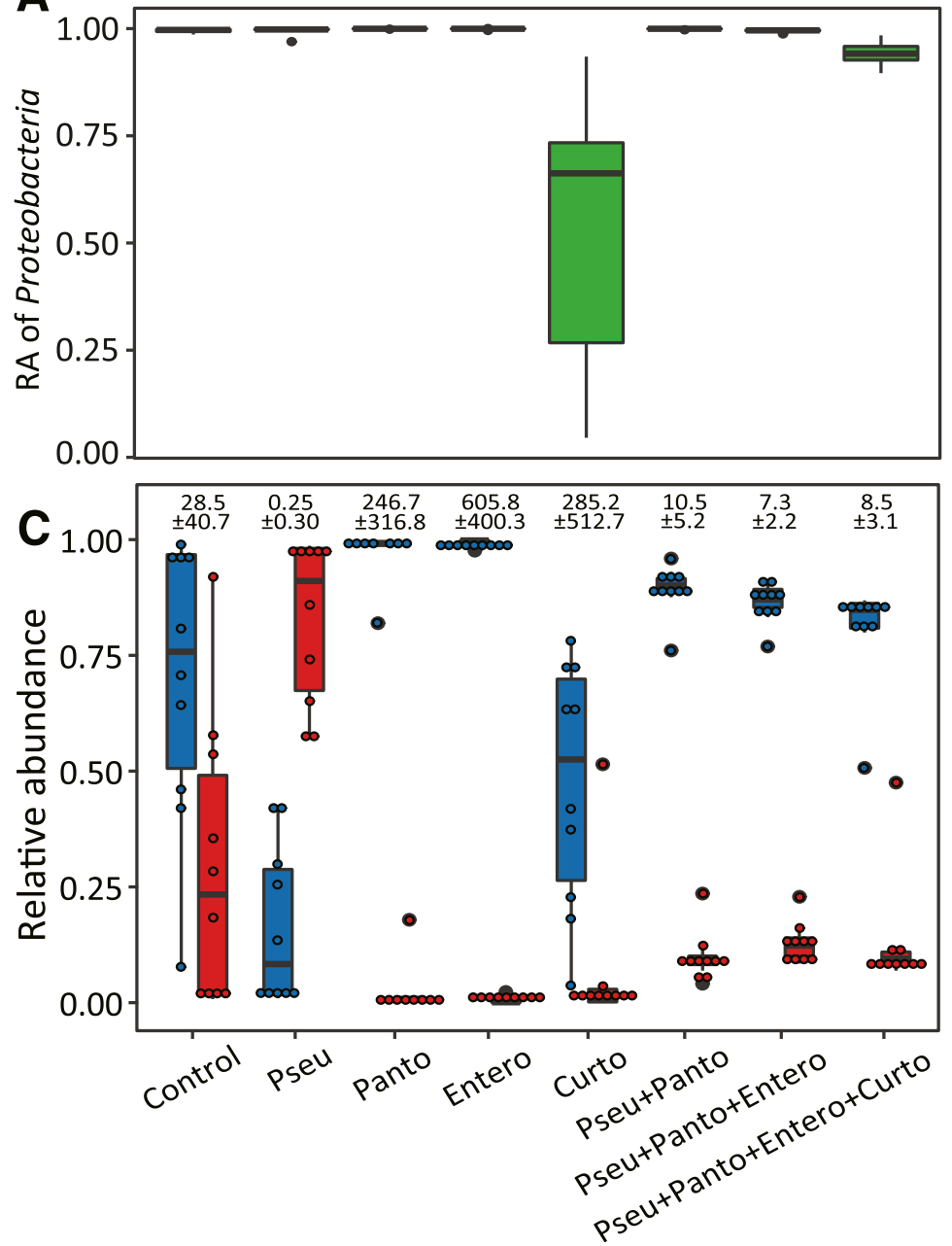

B

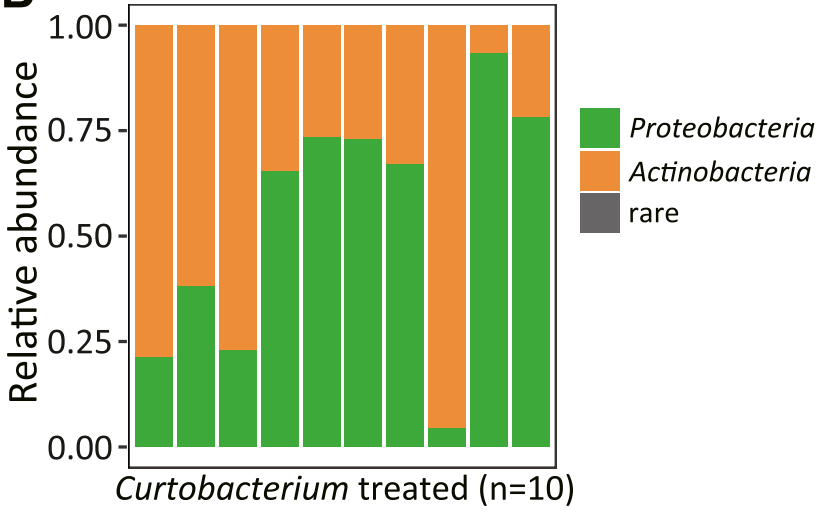

Enterobacteriaceae

Pseudomonadaceae

Fig. 3. A, Mean relative abundance (RA) of the predominant bacterial phylum Proteobacteria. B, Relative abundance of the phyla Actinobacteria and Proteobacteria on individual flowers manipulated with Curtobacterium. The category "rare" represents the sum of the remaining taxa. C, Relative abundance of the two predominant bacterial families upon water and microbial treatments. Each dot represents a single flower sample. The ratio of Enterobacteriaceae to Pseudomonadaceae is presented on the top of each treatment with standard division. Statistical significance was determined by analysis of variance comparisons of means, employing a posthoc Tukey-Cramer test for multiple comparisons. Control: flowers sprayed with sterile $\mathrm{H}_{2} \mathrm{O}$, Pseu: Pseudomonas spp., Panto: Pantoea spp., Entero: Enterobacter spp., and Curto: Curtobacterium spp. 
In vitro antagonistic test of selected strains against E. amylovora. The selection criteria of the strains tested in this study were not the biocontrol activities but, rather, their representation of different members of the stigma microbiome. Our final goal was to determine the potential mechanisms of disease suppression by the inoculated strains in an in vitro assay. Of all four strains tested, only Pseudomonas showed inhibition against the growth of E. amylovora on the LB agar medium (Fig. 5). Interestingly, despite the antagonistic activity toward E. amylovora, the Pseudomonas strain did not show the highest disease inhibition (Fig. 5). Instead, the Pantoea strain, alone or in a strain mixture, showed the greatest disease suppression (Fig. 5). One potential explanation for the reduced disease incidence could be attributable to niche exclusion of E. amylovora on apple stigmas, which is consistent with previous observations (Giddens et al. 2003). However, when the Pantoea was mixed with Pseudomonas, disease suppression was similar to Pantoea alone or in the other strain mixtures (Fig. 5) but the relative abundance of Pantoea dropped to $>1 \%$ of recovered $16 \mathrm{~S}$ rRNA sequences (Fig. 4). This suggests that the presence of Pantoea is still potentially inhibitory to fire blight development, even when present in low relative abundance. Thus, these data suggest that Pantoea antagonistic activities were not apparent on LB media and would not have been predicted from the relative abundance of Pantoea-related sequences (particularly in the Pseudomonas-Pantoea mixture). These data highlight the importance of monitoring the microbiome in situ to assess the complex interactions that occur between members and how they may interact with outcomes such as disease suppression.

\section{DISCUSSION}

In this study, we demonstrated that the microbiome structure can be altered by artificial inoculation, which resulted in attenuated disease occurrence. Many trials in manipulation of the microbiome with the intention to influence host-microbe interactions, suppress diseases, or promote host-beneficial traits have been attempted (Brugman et al. 2018). The outcomes of such efforts vary significantly, and are highly influenced by the host environment, nutrient stage, and manipulation approaches (Berg and Koskella 2018; Brugman et al. 2018). Unlike many host environments, flowers are nutrient rich but also directly exposed to the natural environment, providing an easy access for manipulation. Compared with some environments such as the root microbiome that require a long-term, steadfast microbiome intervention to produce beneficial effects (Bakker et al. 2013), flowers have a short window of bloom; thus, the microbiome manipulation could be short-term yet the influence on disease control and plant health could still be significant.

Taxonomic diversity is an important measure of microbiome structure and function (Hu et al. 2016; Trivedi et al. 2012). Our data suggest that the diversity of the microbial community on the apple stigma is not directly correlated with the occurrence of fire blight. For example, similar taxonomic diversity was observed on flowers treated with strains of Enterobacter and a mixture of Pseudomonas and Pantoea, although the disease occurrence of such treatments differed (Figs. 1 and 2B). Furthermore, among treatments of double-, triple-, and quadruple-strain mixtures, although the diversity gradually increased in accordance with the increasing number of strains, no significant difference in their disease protection was observed (Figs. 1 and 2B; Supplementary Fig. S3). In other plant hosts such as tomato, the level of disease protection ability of a constructed bacterial community was also found to be independent of overall bacterial diversity (Berg and Koskella 2018). Thus, the hypothesis that a more complex microbial inoculation would result in better disease suppression was not supported. Here, our data point to a trend that treatments with Pantoea generally lead to better disease suppression than treatments that didn't include Pantoea.

Natural flowers harbored varied microbiome communities, resulting in unique structures. The largest effect of the microbial treatments on the microbiome was a reduction in the interindividual variability, with the exception of treatments with Pseudomonas and Curtobacterium. In particular, microbial treatments using Pseudomonas-Pantoea-based double-, triple-, and quadruple-strain

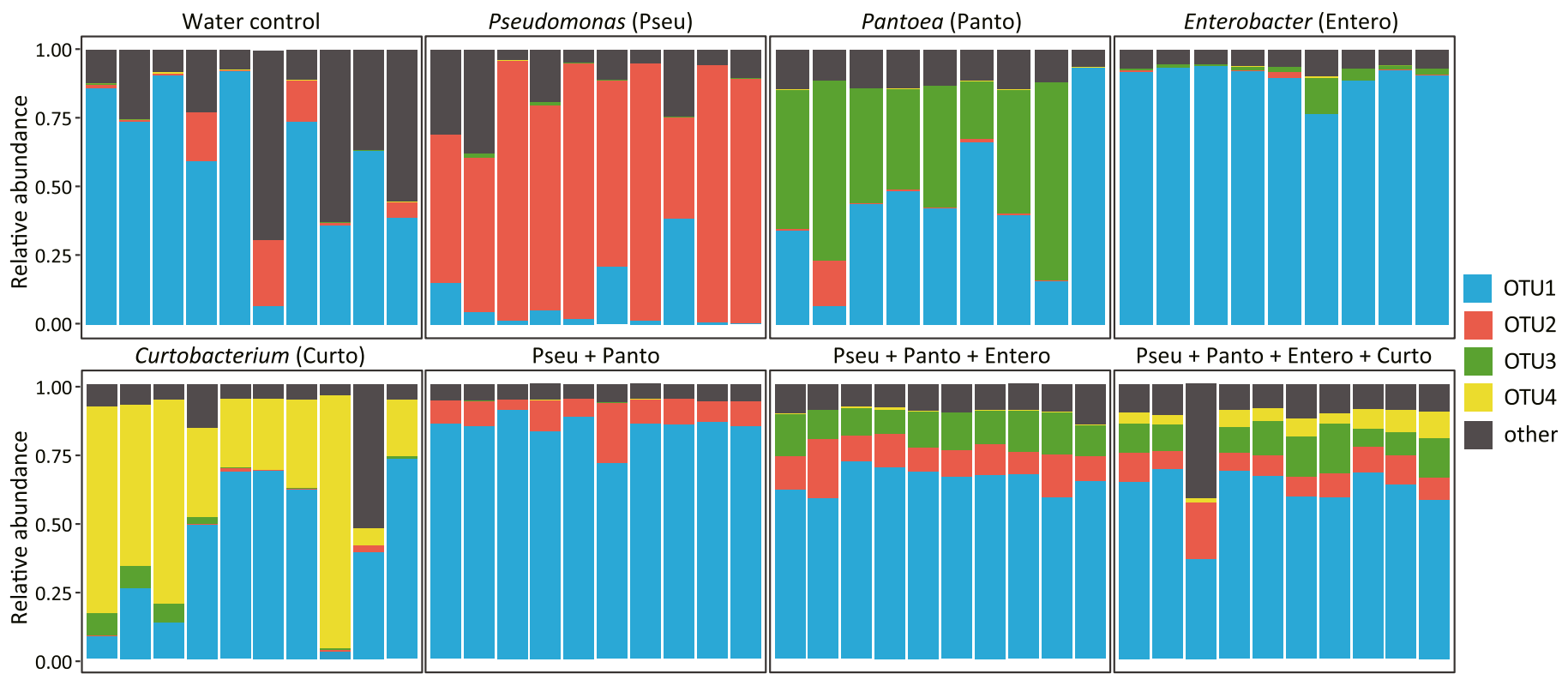

Fig. 4. Identification of inoculated strains in the microbiome. Each column represents the microbial composition of a single flower. Four operational taxonomic units (OTUs) with 100\% sequence identity to the inoculated strains are displayed, and the category "other" represents the sum of the remaining taxa. OTU1: Enterobacter sp. strain CT-1341, OTU2: Pseudomonas sp. strain CT-1059, OTU3: Pantoea sp. strain CT-1039, and OTU4: Curtobacterium sp. strain CT-1342. 
mixtures resulted in the smaller distances between samples in the NMDS clustering (Fig. 2A) and the similarity in the $\mathrm{R}_{E / P}$ between replicate flowers (Fig. 3C). This is important because it suggests that there should also be more uniformity in any phenotypic effects induced by the microbiome. A common observation is that only a proportion of flowers sprayed with E. amylovora in the field will later go on to develop fire blight (Cui et al. in press; Sundin et al. 2009). Similarly, many biocontrol strains have been described with excellent antagonistic potential under in vitro or in planta conditions; however, they present inconsistent effectiveness and year-to- year variation under field conditions (Johnson and Stockwell 1998; Sundin et al. 2009). One potential reason for the variability observed at the individual flower level and for interannual irregularity could be the large interindividual disparity in microbiome structure between flowers. Thus, by shifting the microbiome to a state that is predictable, repeatable, and stable and also induces disease resistance, we may be able to manage such variations. Although none of the manipulations employed here attained the same disease reduction as the streptomycin treatment, they did produce a highly conserved microbiome structure (Fig. 4) and up to an approximately
A

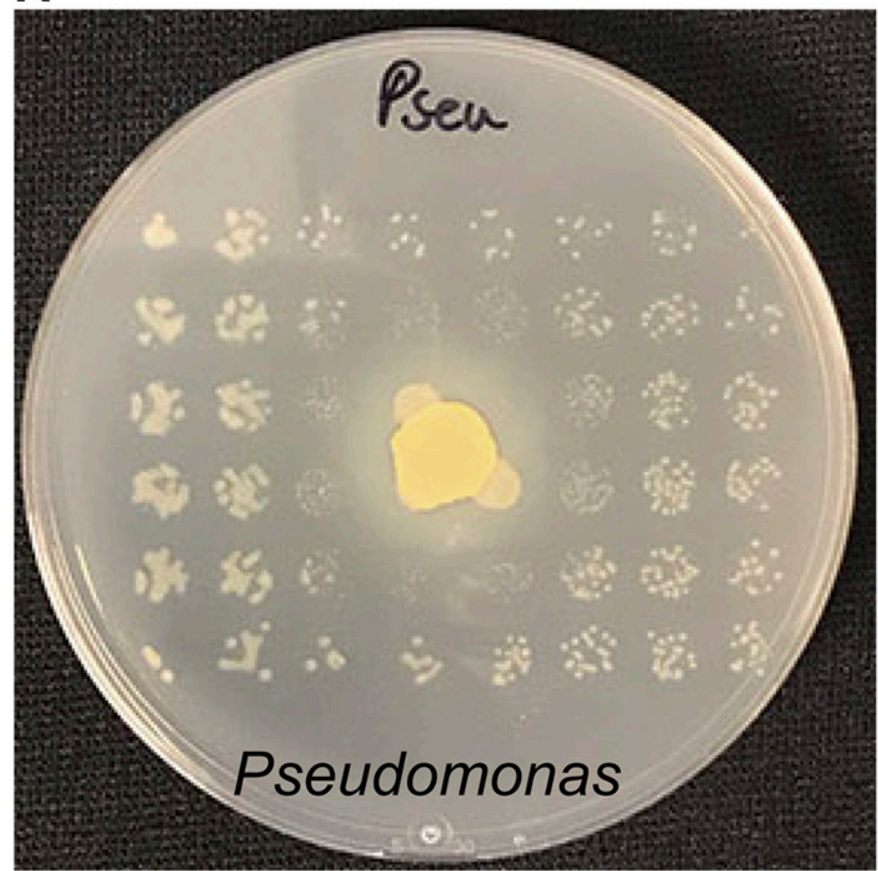

C

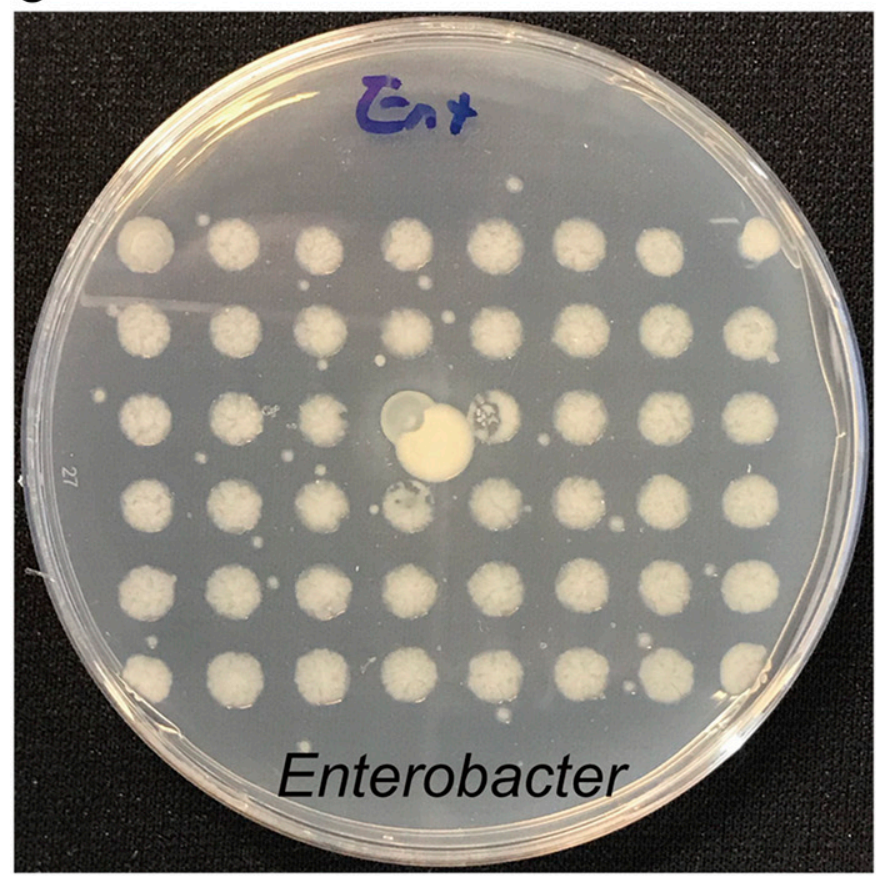

B

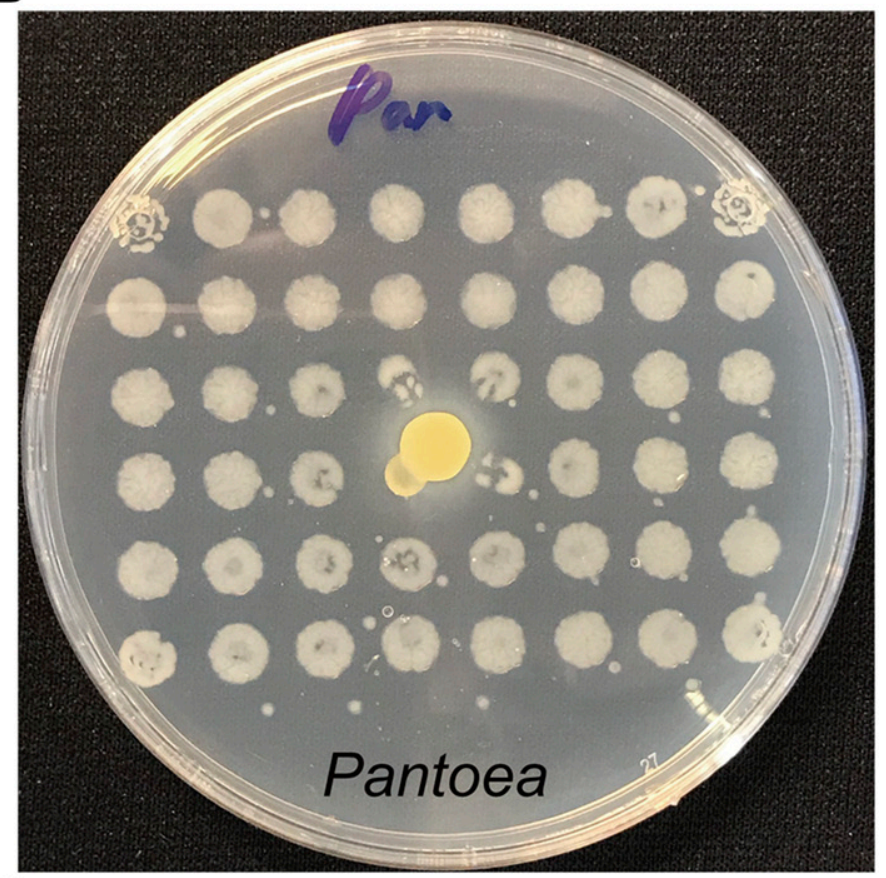

D

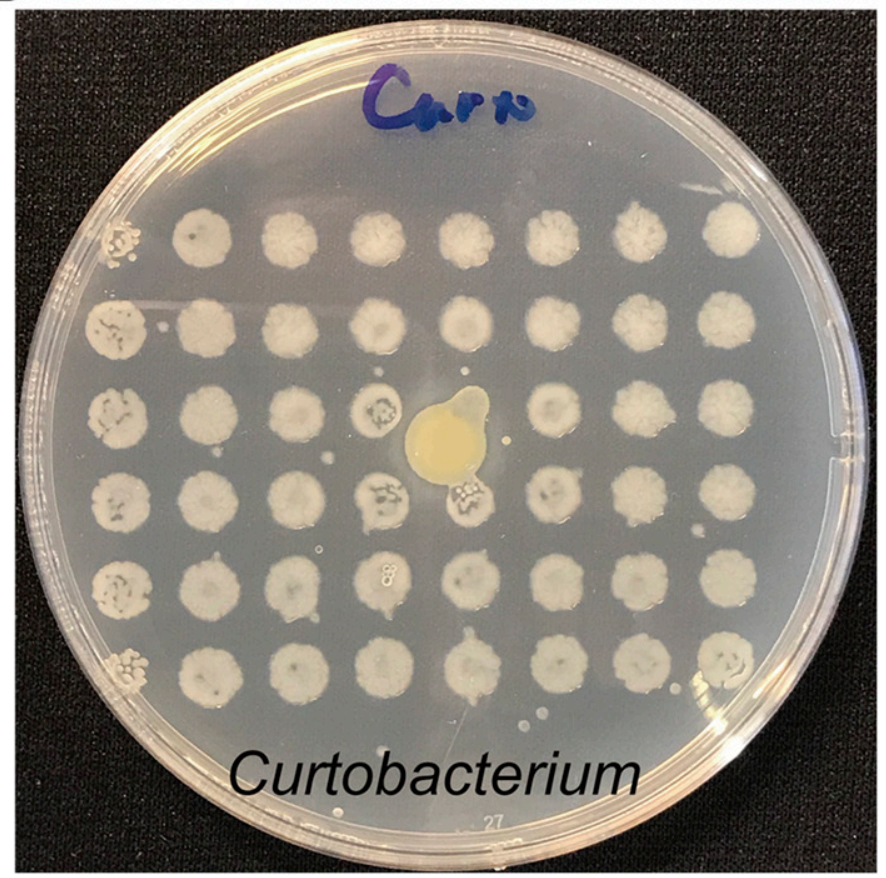

Fig. 5. In vitro antagonism of strain A, Pseudomonas spp.; B, Pantoea spp.; C, Enterobacterspp.; and D, Curtobacterium spp. against Erwinia amylovora strain 110. Tested strains were precultured on Lysogen Broth agar plates for $48 \mathrm{~h}$. E. amylovora 110 was inoculated onto the plates using a prong dipped in bacterial suspension. 
$45 \%$ decrease fire blight (Fig. 1). This suggests that a microbiomeinformed treatment strategy may be a viable path to controlling plant diseases.

Recent work trying to predict community assembly from first principals found that complex communities seeded on a glucose minimal medium predictably adopted a state dominated by members of the families Enterobacteriaceae and Pseudomonadaceae in a phenomenon termed "emergent simplicity" (Goldford et al. 2018). The predictability of this assembly points to certain metabolic rules, based on cross-feeding, determining the final state of the microbiome (Estrela et al. 2020; Treves et al. 1998; Turner et al. 1996). Here, we observed a similar phenomenon in a natural environment. The ratio of families and the particular members of the microbiome can be seeded early in the development of the stigma microbiome, leading to a predictable community structure. Untangling the metabolic interactions between community members will better inform mechanisms by which the microbiome can be shifted to potentially beneficial states.

One limitation of this study is that some LB ingredients were sprayed to flowers during the microbial treatments, because the overnight culture of the bacterial inoculum was directly diluted in water prior to application on flowers. This limitation is caused by the need for large volumes of inoculum for field application and the unavailability of a large centrifuge that can process litters of bacterial culture to remove the media ingredients. The dilution factor for the bacterial cultures was approximately 25 -fold and the media content was largely depleted after bacterial growth; thus, the amount of nutrients remaining in the bacterial inoculum was likely minimal. However, the leftover nutrients and potential secondary metabolites were not present in the water controls, which could account for some of the observed differences. Thus, during the analysis of the microbiome data, we only directly compared the different microbial treatments. The microbiome structure, composition, and diversity in water controls were provided as a reference for the development of the microbiome in the absence of manipulation.

In summary, our data point to a common state of the stigma microbiome being dominated by bacteria in two families, Enterobacteriaceae and Pseudomonadaceae. However, a probiotic spray in the field can determine the particular members that make up this state. Treating flowers with certain microbial members, particularly a strain of Pantoea, was associated with disease suppression. Multispecies inoculations showed that there are complex interactions between the treatment strains but there is some evidence that a more complex inoculation can enhance disease resistance. These data will inform both treatments for fire blight as well as the ecological principals and interactions that determine the structure and function of the flower microbiome.

\section{ACKNOWLEDGMENTS}

We thank R. Cecarelli, R. Hannan, and M. McHill from the Connecticut Agricultural Experiment Station Lockwood Farm for their excellent technical support.

\section{LITERATURE CITED}

Ait Bahadou, S., Ouijja, A., Karfach, A., Tahiri, A., and Lahlali, R. 2018. New potential bacterial antagonists for the biocontrol of fire blight disease (Erwinia amylovora) in Morocco. Microb. Pathog. 117:7-15.

Aleklett, K., Hart, M., and Shade, A. 2014. The microbial ecology of flowers: An emerging frontier in phyllosphere research. Botany 92:253-266.

Bakker, P. A., Berendsen, R. L., Doornbos, R. F., Wintermans, P. C., and Pieterse, C. M. 2013. The rhizosphere revisited: Root microbiomics. Front. Plant Sci. 4:165.
Berendsen, R. L., Vismans, G., Yu, K., Song, Y., de Jonge, R., Burgman, W. P., Burmølle, M., Herschend, J., Bakker, P. A., and Pieterse, C. M. 2018. Disease-induced assemblage of a plant-beneficial bacterial consortium. ISME J. 12:1496-1507.

Berg, M., and Koskella, B. 2018. Nutrient- and dose-dependent microbiomemediated protection against a plant pathogen. Curr. Biol. 28: 2487-2492.e3.

Bonaterra, A., Cabrefiga, J., Camps, J., and Montesinos, E. 2007. Increasing survival and efficacy of a bacterial biocontrol agent of fire blight of rosaceous plants by means of osmoadaptation. FEMS Microbiol. Ecol. 61:185-195.

Brugman, S., Ikeda-Ohtsubo, W., Braber, S., Folkerts, G., Pieterse, C. M. J., and Bakker, P. 2018. A Comparative review on microbiota manipulation: Lessons from fish, plants, livestock, and human research. Front. Nutr. 5:80.

Brunings, A. M., and Gabriel, D. W. 2003. Xanthomonas citri: Breaking the surface. Mol. Plant Pathol. 4:141-157.

Chen, X.-H., Scholz, R., Borriss, M., Junge, H., Mögel, G., Kunz, S., and Borriss, R. 2009. Difficidin and bacilysin produced by plant-associated Bacillus amyloliquefaciens are efficient in controlling fire blight disease. J. Biotechnol. 140:38-44.

Crawford, B. C., and Yanofsky, M. F. 2008. The formation and function of the female reproductive tract in flowering plants. Curr. Biol. 18:R972-R978.

Cui, Z., Huntley, R., Zeng, Q., and Steven, B. Temporal and spatial dynamics in the apple flower microbiome in the presence of the phytopathogen Erwinia amylovora. ISME J. In press. doi.org/10.1038/s41396-020-00784-y

Dixon, P. 2003. VEGAN, a package of R functions for community ecology. J. Veg. Sci. 14:927-930.

Estrela, S., Vila, J. C. C., Lu, N., Bajic, D., Rebolleda-Gomez, M., Chang, C.-Y., and Sanchez, A. 2020. Metabolic rules of microbial community assembly. BioRxiv. doi:10.1101/2020.03.09.984278

Giddens, S. R., Houliston, G. J., and Mahanty, H. K. 2003. The influence of antibiotic production and pre-emptive colonization on the population dynamics of Pantoea agglomerans (Erwinia herbicola) Eh1087 and Erwinia amylovora in planta. Environ. Microbiol. 5:1016-1021.

Goldford, J. E., Lu, N., Bajic, D., Estrela, S., Tikhonov, M., Sanchez-Gorostiaga, A., Segre, D., Mehta, P., and Sanchez, A. 2018. Emergent simplicity in microbial community assembly. Science 361:469-474.

Hu, J., Wei, Z., Friman, V. P., Gu, S. H., Wang, X. F., Eisenhauer, N., Yang, T. J., Ma, J., Shen, Q. R., Xu, Y. C., and Jousset, A. 2016. Probiotic diversity enhances rhizosphere microbiome function and plant disease suppression. MBio 7:e01790-16.

Johnson, K. B., and Stockwell, V. O. 1998. Management of fire blight: A case study in microbial ecology. Annu. Rev. Phytopathol. 36:227-248.

Johnson, K. B., and Stockwell, V. O. 2000. Biological control of fire blight. Pages 319-338 in: Fire Blight, the Disease and its Causal Agent, Erwinia amylovora. J. L. Vanneste, ed. CAB International, Wallingford, U.K.

Johnson, K. B., and Temple, T. N. 2013. Evaluation of strategies for fire blight control in organic pome fruit without antibiotics. Plant Dis. 97:402-409.

Leben, C. 1965. Epiphytic microorganisms in relation to plant disease. Annu. Rev. Phytopathol. 3:209-230.

McGhee, G. C., Guasco, J., Bellomo, L. M., Blumer-Schuette, S. E., Shane, W. W., Irish-Brown, A., and Sundin, G. W. 2011. Genetic analysis of streptomycin-resistant $\left(\mathrm{Sm}^{\mathrm{R}}\right)$ strains of Erwinia amylovora suggests that dissemination of two genotypes is responsible for the current distribution of $\mathrm{Sm}^{\mathrm{R}}$ E. amylovora in Michigan. Phytopathology 101:182-191.

Mikiciński, A., Puławska, J., Molzhigitova, A., and Sobiczewski, P. 2020. Bacterial species recognized for the first time for its biocontrol activity against fire blight (Erwinia amylovora). Eur. J. Plant Pathol. 156:257-272.

Morella, N. M., Zhang, X., and Koskella, B. 2019. Tomato seed-associated bacteria confer protection of seedlings against foliar speck caused by Pseudomonas syringae. Phytobiomes J. 3:177-190.

Purahong, W., Orrù, L., Donati, I., Perpetuini, G., Cellini, A., Lamontanara, A., Michelotti, V., Tacconi, G., and Spinelli, F. 2018. Plant microbiome and its link to plant health: Host species, organs and Pseudomonas syringae pv. actinidiae infection shaping bacterial phyllosphere communities of kiwifruit plants. Front. Plant Sci. 9:1563.

Pusey, P. L. 1997. Crab apple blossoms as a model for research on biological control of fire blight. Phytopathology 87:1096-1102.

Pusey, P. L. 2000. The role of water in epiphytic colonization and infection of pomaceous flowers by Erwinia amylovora. Phytopathology 90:1352-1357.

Pusey, P. L., Stockwell, V. O., and Mazzola, M. 2009. Epiphytic bacteria and yeasts on apple blossoms and their potential as antagonists of Erwinia amylovora. Phytopathology 99:571-581.

Pusey, P. L., Stockwell, V. O., Reardon, C. L., Smits, T. H. M., and Duffy, B. 2011. Antibiosis activity of Pantoea agglomerans biocontrol strain E325 
against Erwinia amylovora on apple flower stigmas. Phytopathology 101: 1234-1241.

Quast, C., Pruesse, E., Yilmaz, P., Gerken, J., Schweer, T., Yarza, P., Peplies, J., and Glöckner, F. O. 2012. The SILVA ribosomal RNA gene database project: Improved data processing and web-based tools. Nucleic Acids Res. 41:D590-D596.

Rognes, T., Flouri, T., Nichols, B., Quince, C., and Mahe, F. 2016. VSEARCH: A versatile open source tool for metagenomics. PeerJ 4:e2584.

Schloss, P. D., Westcott, S. L., Ryabin, T., Hall, J. R., Hartmann, M., Hollister, E. B., Lesniewski, R. A., Oakley, B. B., Parks, D. H., Robinson, C. J., Sahl, J. W., Stres, B., Thallinger, G. G., Van Horn, D. J., and Weber, C. F. 2009. Introducing mothur: Open-source, platform-independent, communitysupported software for describing and comparing microbial communities. Appl. Environ. Microbiol. 75:7537-7541.

Sinclair, L., Osman, O. A., Bertilsson, S., and Eiler, A. 2015. Microbial community composition and diversity via $16 \mathrm{~S}$ rRNA gene amplicons: Evaluating the Illumina platform. PLoS One 10:e0116955.

Spinelli, F., Ciampolini, F., Cresti, M., Geider, K., and Costa, G. 2005. Influence of stigmatic morphology on flower colonization by Erwinia amylovora and Pantoea agglomerans. Eur. J. Plant Pathol. 113:395-405.

Steiner, P. W. 1990. Predicting apple blossom infections by Erwinia amylovora using the MARYBLYT model. Acta Hortic. 273:139-148.

Steven, B., Huntley, R., and Zeng, Q. 2018. The influence of flower anatomy and apple cultivar on the apple flower phytobiome. Phytobiomes J. 2:171-179.

Stockwell, V. O., Johnson, K. B., Sugar, D., and Loper, J. E. 2010. Control of fire blight by Pseudomonas fluorescens A506 and Pantoea vagans C9-1 applied as single strains and mixed inocula. Phytopathology 100:1330-1339.

Stockwell, V. O., Johnson, K. B., Sugar, D., and Loper, J. E. 2011. Mechanistically compatible mixtures of bacterial antagonists improve biological control of fire blight of pear. Phytopathology 101:113-123.

Sundin, G. W., Castiblanco, L. F., Yuan, X., Zeng, Q., and Yang, C. H. 2016. Bacterial disease management: Challenges, experience, innovation and future prospects. Mol. Plant Pathol. 17:1506-1518.

Sundin, G. W., Werner, N. A., Yoder, K. S., and Aldwinckle, H. S. 2009. Field evaluation of biological control of fire blight in the eastern United States. Plant Dis. 93:386-394.
Thomson, S. 1986. The role of the stigma in fire blight infections. Phytopathology 76:476-482.

Treves, D. S., Manning, S., and Adams, J. 1998. Repeated evolution of an acetate-crossfeeding polymorphism in long-term populations of Escherichia coli. Mol. Biol. Evol. 15:789-797.

Trivedi, P., He, Z., Van Nostrand, J. D., Albrigo, G., Zhou, J., and Wang, N. 2012. Huanglongbing alters the structure and functional diversity of microbial communities associated with citrus rhizosphere. ISME J. 6: 363-383.

Turner, P. E., Souza, V., and Lenski, R. E. 1996. Tests of ecological mechanisms promoting the stable coexistence of two bacterial genotypes. Ecology 77: 2119-2129.

van der Zwet, T., Orolaza-Halbrendt, N., and Zeller, W. 2012. Fire Blight History, Biology, and Management. American Phytopathological Society, St. Paul, MN, U.S.A.

Vanneste, J., Ede, F., and Yu, J. 1995. Influence of delayed inoculation on efficacy of Erwinia herbicola and streptomycin to control fire blight. Biol. Cult. Tests Control Plant Dis. 10:46.

Wang, Q., Garrity, G. M., Tiedje, J. M., and Cole, J. R. 2007. Naive Bayesian classifier for rapid assignment of rRNA sequences into the new bacterial taxonomy. Appl. Environ. Microbiol. 73:5261-5267.

Weller, D. M., Raaijmakers, J. M., Gardener, B. B., and Thomashow, L. S. 2002. Microbial populations responsible for specific soil suppressiveness to plant pathogens. Annu. Rev. Phytopathol. 40:309-348.

Westcott, S. L., and Schloss, P. D. 2017. OptiClust, an improved method for assigning amplicon-based sequence data to operational taxonomic units. MSphere 2:e00073-17.

Wickham, H. 2016. ggplot2: Elegant Graphics for Data Analysis. SpringerVerlag, New York, NY, U.S.A.

Wilson, M., Epton, H. A. S., and Sigee, D. C. 1992. Biological control of fire blight of hawthorn (Crataegus monogyna) with fluorescent Pseudomonas spp. under protected conditions. J. Phytopathol. 136:16-26.

Xin, X.-F., and He, S. Y. 2013. Pseudomonas syringae pv. tomato DC3000: A model pathogen for probing disease susceptibility and hormone signaling in plants. Annu. Rev. Phytopathol. 51:473-498. 